\title{
An overview of minimal incisional ptosis correction: A surgeon's perspective
}

\section{Tae Joo Ahn}

SU:I Plastic Surgery, Seoul, Korea

This article was presented at Aesthetic Plastic Surgery 2019 on April 6-7, 2019, in Seoul, Korea.
People increasingly prefer fast and convenient methods for aesthetic procedures in busy modern society. Therefore, physicians and patients increasingly desire to improve cases of mild ptosis of the eyes in a simpler way. The purpose of this review is to organize the surgical methods of minimal incisional ptosis correction that the author has developed to satisfy this need and to examine the indications of each method and its advantages and disadvantages. The basic technique is a triangular single-knot stitch method using five points. Additionally, the method of applying a special loop (tucking the Müller muscle by pulling the conjoint fascial sheath) and the method of combining a non-incisional method with making a loop will be explained herein.

Keywords Blepharoptosis / Blepharoplasty / Eyelid / Muscle

\section{INTRODUCTION}

As modern life becomes busier, patients increasingly desire simple methods for aesthetic procedures that allow a speedy recovery. The demand for filler procedures, Botox injections, thread lifting, and similar procedures is continuously increasing, and patients' preference for simpler and easier surgical procedures is a growing trend.

The eyes are an important component of facial expression. In supratarsal crease surgery (double-fold surgery), the stitch method is increasingly preferred to the incisional method. Additionally, for correction of mild ptosis, more and more patients request the stitch method, which involves a less severe wound and allows a quick recovery, and minimal incisional ptosis correction fits those needs. This review aims to organize the author's methods for minimal incisional ptosis correction and to examine the indications of each method and its advantages and disadvantages.

Received: Aug 16, 2019 Revised: Oct 1, 2019 Accepted: Oct 2, 2019 Correspondence: Tae Joo Ahn SU:I Plastic Surgery, 480 Gangnam-daero, Gangnam-gu, Seoul 06120, Korea

Tel: +81-2-535-6688, Fax: +82-2-535-8580, E-mail: cmcanti@hanmail.net

Copyright @ 2020 The Korean Society for Aesthetic Plastic Surgery.

This is an Open Access article distributed under the terms of the Creative Commons Attribution Non-Commercial License (https://creativecommons.org/licenses/by-nc/4.0/) which permits unrestricted non-commercial use, distribution, and reproduction in any medium, provided the original work is properly cited. www.e-aaps.org

\section{OPERATIVE TECHNIQUES}

Minimal incisional supratarsal crease surgery: The triangular single-knot stitch method

The author usually uses the stitch method with a triangular suture. If the number of triangles is increased, the force pulling on the eyelid skin becomes stronger without serious tissue swelling. Thus, this technique can be applied to relatively thick skin and to cases where the eyelid fold has become undone after the primary incision method (Fig. 1).

A double eyelid appears as the skin of the anterior lamella is pulled in the posterior direction. This pulling force, referred to as the backward vector, increases with the number of triangles. Additionally, given the same width, the area where soft tissue undergoes strangulation does not change even if the number of triangles increases. Therefore, the force used to fold skin can be increased without long-lasting swelling (Fig. 1B-D). Another advantage of the triangular stitch method is that even if the biting effect of the suture digging into the skin occurs, it usually affects the corners of the triangles. Therefore, the overall pulling force is well maintained and irritation of the conjunctiva is reduced. In other words, compared to rectangular loops, a trigonal suture can maintain a double eyelid more strongly with less swelling and reduce the irritation of the conjunctiva. 

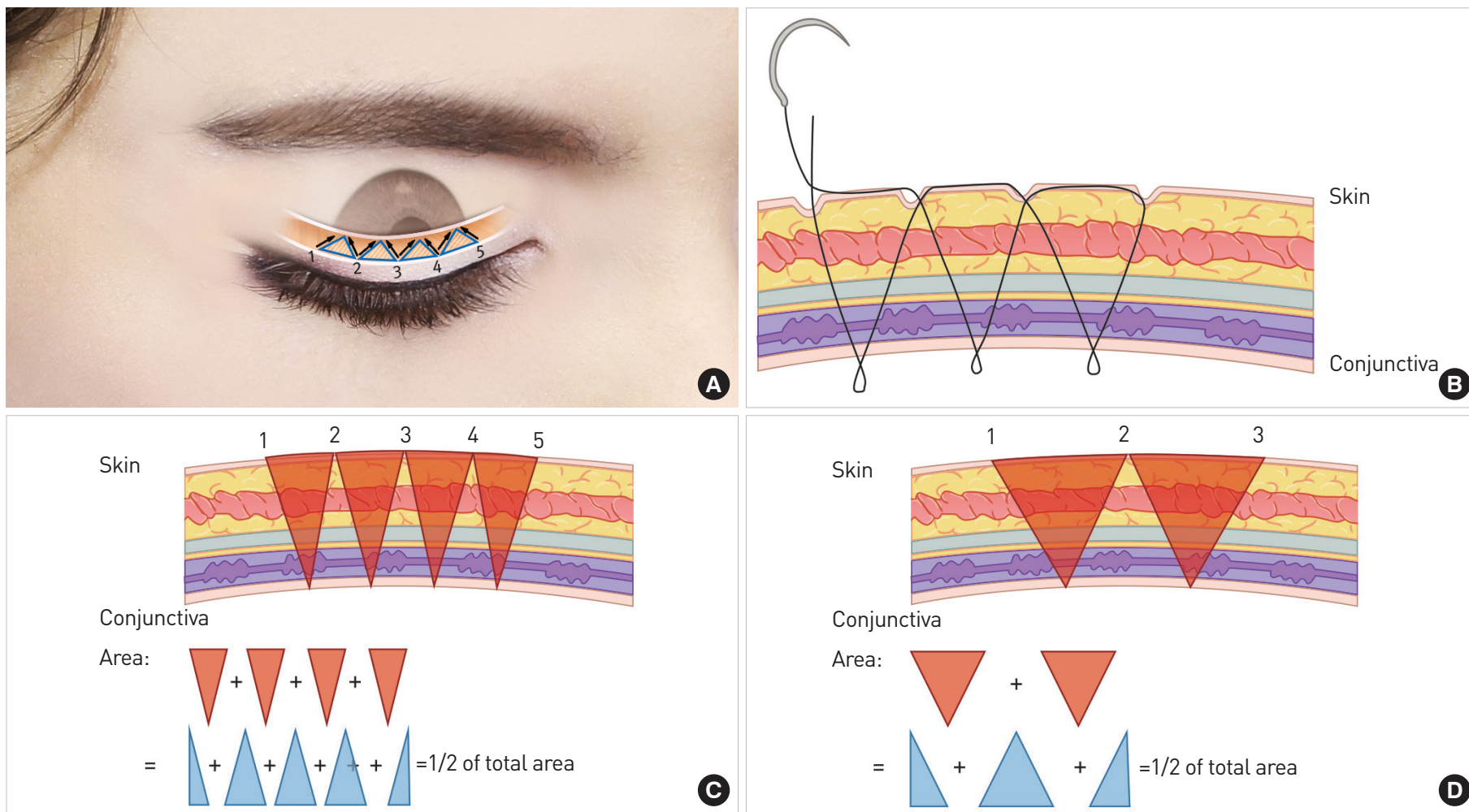

Fig. 1. Triangular single-knot stitch method. (A) Triangular single-knot stitch method and a three-dimensional image. (B) Cross-sectional image of the triangular single-knot stitch method. The method of making a triangle through five slits (C) can yield a stronger force than when a triangle is made through three slits (D). If the number of points is increased with the same width, as the area where soft tissue undergoes strangulation is the same, longer-lasting swelling does not occur even though the force is increased by the greater number of points.

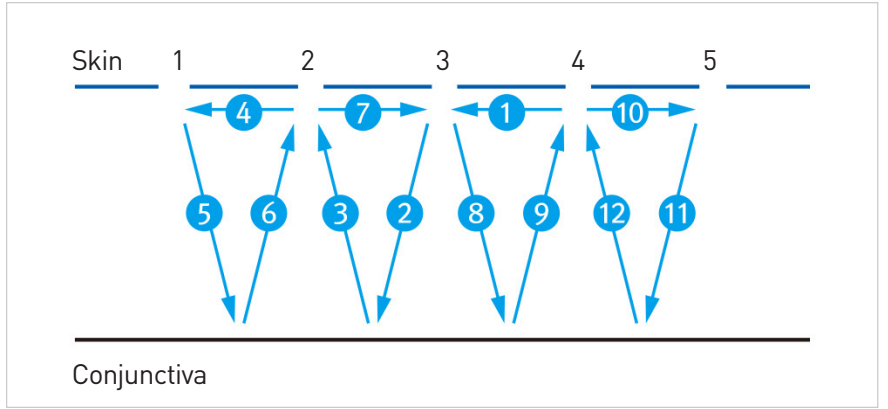

Fig. 2. Order of the 5-point method.

\section{The 5-point method}

Five small slits are made using a No. 11 blade along the expected double eyelid line, and they are numbered 1 to 5 from the medial side to the lateral side. The suture is passed just below the skin from No. 4 to No. 3 and is then passed through the conjunctiva from No. 3 to No. 2. Passing below the skin from No. 2 to No. 1 and through the conjunctiva from No. 1 to No. 2 is repeated, thereby completing the triangular single-knot stitch method (Fig. 2) [1-3].

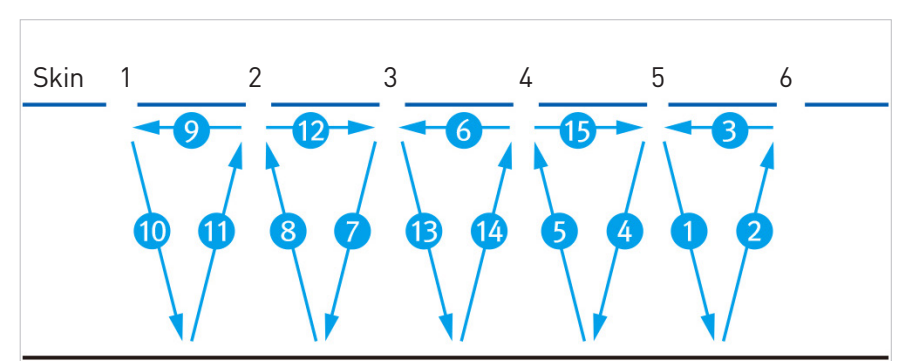

Conjunctiva

Fig. 3. Order of the 6-point method.

\section{The 6-point method}

If a patient has relatively thick skin or the eyelid fold comes undone after the 5-point incision method is used, the number of points can be increased to six. Similar to the 5-point method, six slits are made from the medial side to the lateral side numbering 1 to 6 . The suture passes through the conjunctiva from No. 5 to No. 6, below the skin from No. 6 to No. 5, again through the conjunctiva from No. 5 to No. 4 , below the skin from No. 4 to No. 3, and again out through the conjunctiva from No. 3 to No. 2. If the suture goes below the skin from No. 2 to No. 1, through the conjunctiva from No. 1 to 


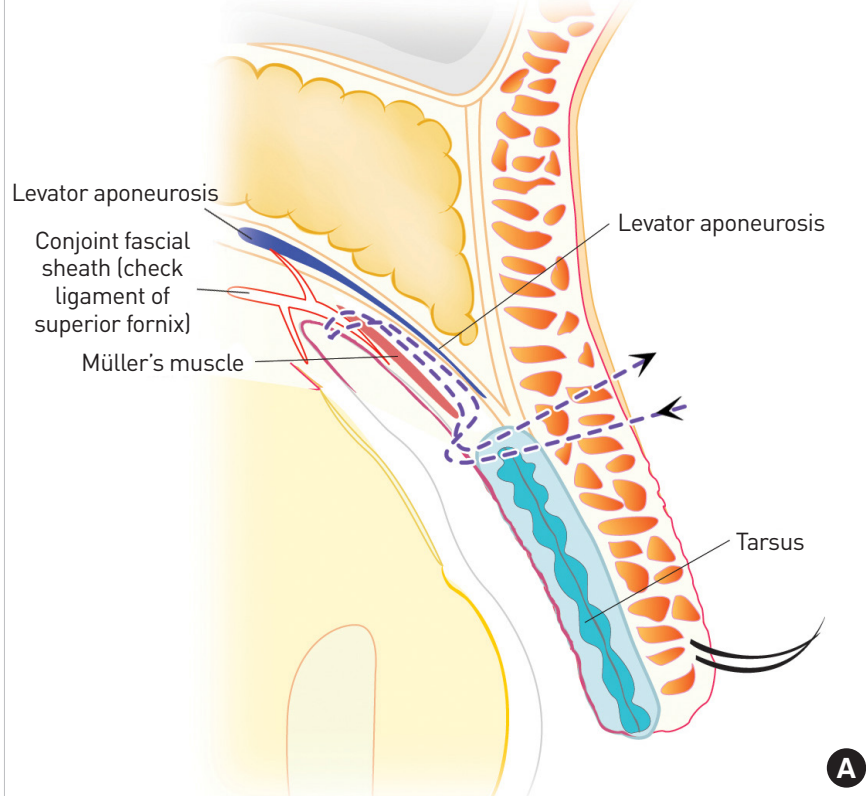

No. 2, below the skin from No. 2 to No. 3 , through the conjunctiva from No. 3 to No. 4 , and below the skin from No. 4 to No. 5, the single-knot stitch method is completed. Lastly, when the knot is made, the force should be adjusted so that the knot can be pulled into the subcutaneous tissue. As such, by increasing the number of points, the force used to pull the anterior skin in the posterior direction can be increased (Fig. 3) [3,4].

\section{Conjoint fascial sheath suspension (CFS sling):}

\section{A separate method}

Similar to existing non-incisional methods, after the suture needle emerges from the conjunctiva through the slit in the skin, Müller muscle tucking is done. If the needle ascends in the superior direction beneath the Müller muscle to the CFS and then descends, Müller muscle tucking is achieved. The needle coming from the conjunctival side is pulled out through the skin. By connecting the inlet thread with the outlet thread, a knot is made. A tucking procedure can be done both with one slit and two slits. Only Müller muscle tucking may be done, or a non-incisional method for additionally creating a double eyelid can be added if necessary. When a double eyelid is created with the non-incisional method, the suture can be made by connecting with the suture used during Müller muscle tucking (Fig. 4A).

To maintain stable Müller muscle tucking, the author passes the suture through to the superior border of the tarsal plate below, raises the suture by at least $12 \mathrm{~mm}$ until reaching the CFS, and then pulls it down. Furthermore, the suture for Müller muscle tucking and the conjoint facial sheath sling forms a triangle by descending to the side approximately $4 \mathrm{~mm}$ away from the point where it starts to be pulled up (Fig. 4B).
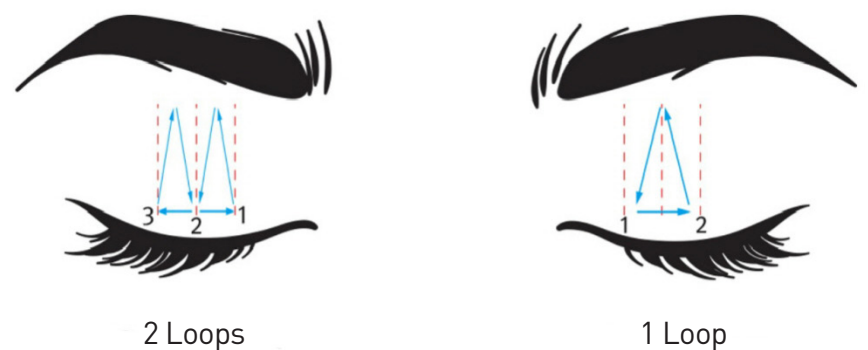

2 Loops

1 Loop

B

Fig. 4. (A) Methods of Müller tucking and conjoint fascial sheath sling. (B) Design with one loop and two loops.

\section{Single-loop method}

If medial or lateral ptosis is not prominent, a loop is made for the eyes to become large with the pupil at the center. With the pupil at the center, a line is drawn perpendicularly from the medial limbus, midpupillary point, and lateral limbus. Point 1 is set between the medial limbus and midpupillary point and point 2 between the midpupillary point and lateral limbus. A 7-0 nylon suture is inserted from point 2 and passed through the conjunctiva on the upper margin of the tarsal plate. Next, a CFS or CFS sling is performed by passing this suture approximately 11 to $15 \mathrm{~mm}$ cephalad and then passing it back caudally to the upper border of the tarsal plate. The suture is next passed anteriorly through point 1 , from points 1 to 2 , the suture is passed through the middle lamella (Fig. 4B right, Supplemental Digital Contents 1 and 2) [3,4].

\section{Double-loop method}

At point 3, running from the skin to the conjunctival side, a CFS sling is made. The suture is next passed anteriorly through point 2 . When moving from point 2 to point 1 , the suture can be passed through a middle layer. At point 1 , the suture is run to the side of the conjunctiva. Next, the CFS sling is made, and then the suture comes out through point 2 . From point 2 to point 3 , the suture is passed through the middle lamella (Fig. 4B left, Supplemental Digital Content 3) [3].

\section{CFS sling with non-incisional double-fold surgery: A continuous method Five-point one-loop method}

In essence, the 5-point method is used. A CFS sling is made at the halfway point when moving from No. 3 to No. 4 (Fig. $5 \mathrm{~A}$ and B) [1,5]. 

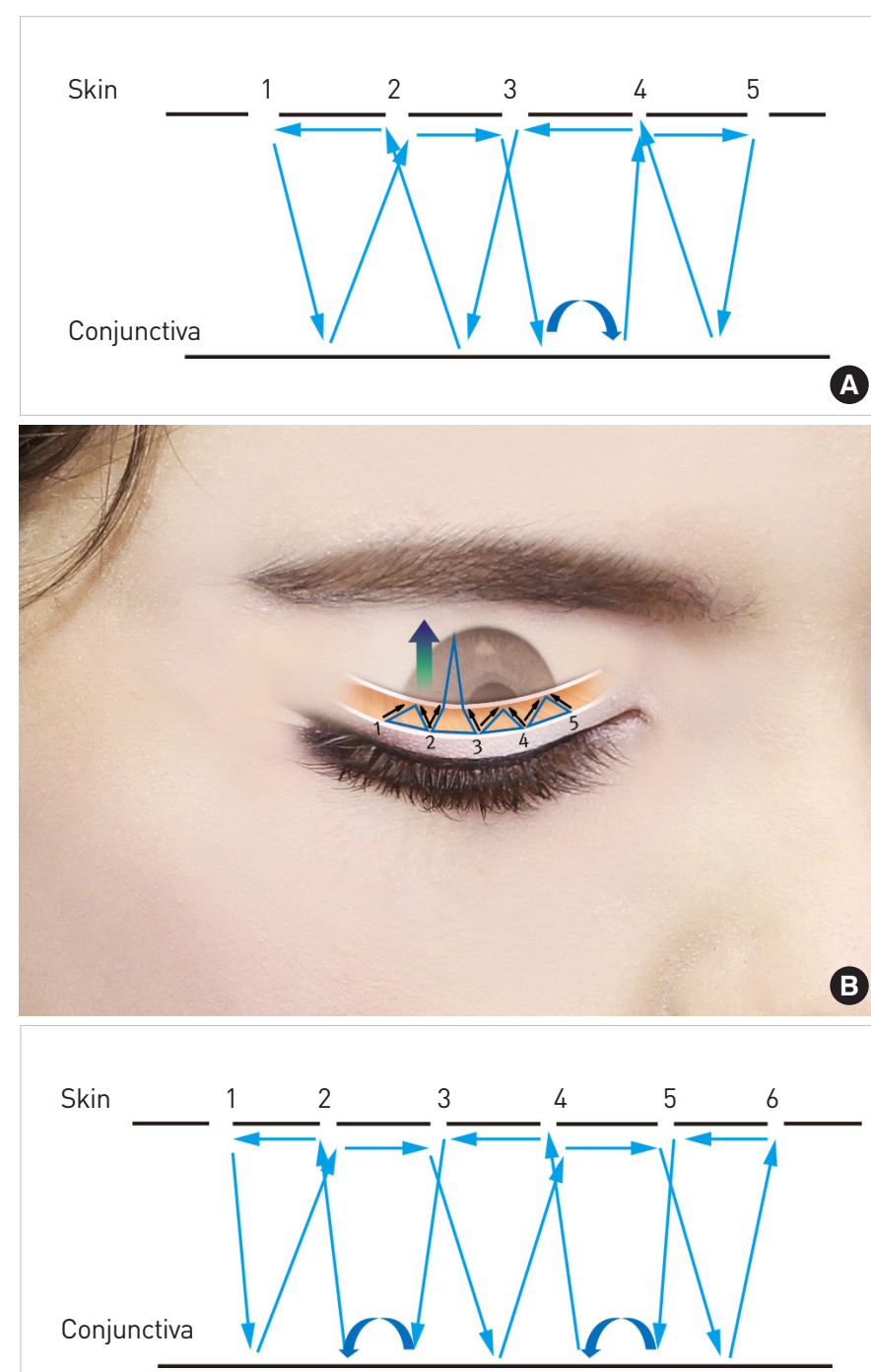

Fig. 5. Cross-sectional image (A) and three-dimensional image of the 5-point one-loop method (B). Image of the 6-point two-loop method (C).

\section{Six-point two-loop method}

Using the 6-point method, a CFS sling is performed at the halfway point when moving from No. 5 to No. 4 and from No. 3 to No. 2 (Fig. 5C, Supplemental Digital Content 4) [5].

\section{Combination of the CFS sling and other methods Combination with skin and muscle excision}

In this technique, one or two loops are added to an existing double eyelid created using an incisional method. It is applied to those who have thick skin or a well-developed orbicularis oculi muscle $(\mathrm{OOM})$; by reducing the factors that interrupt levator action (the force that opposes opening of the eyes), favorable results can be obtained by adding a loop with a CFS sling and Müller muscle tucking [6].

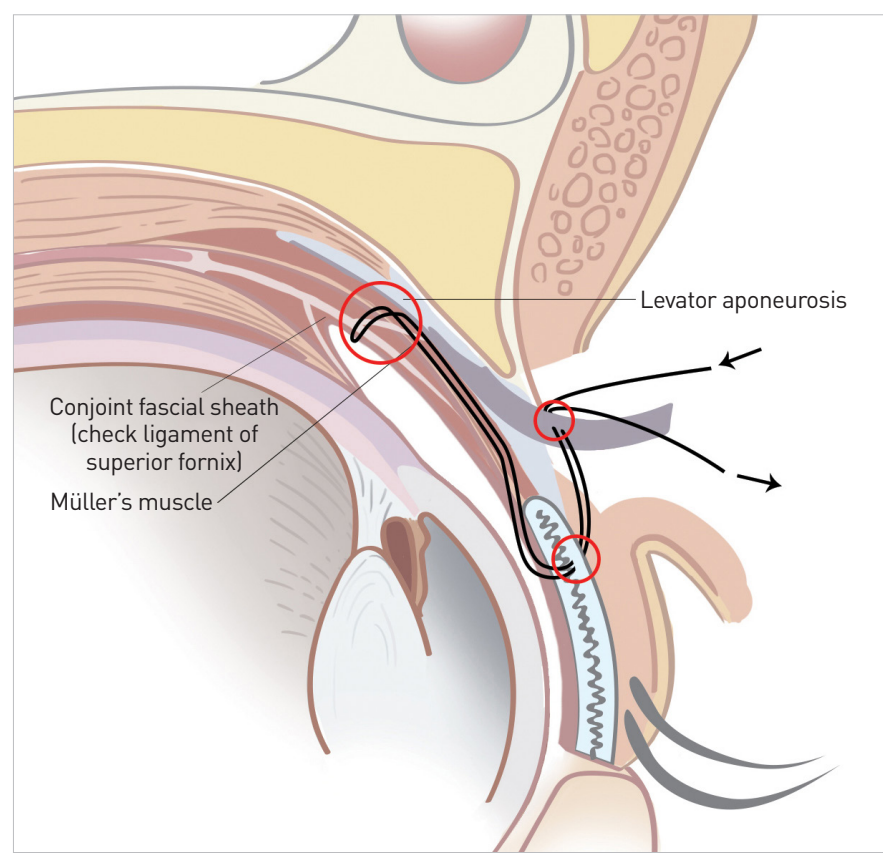

Fig. 6. Method of combining a single-knot double eyelid, Müller tucking, and levator advancement along with skin and orbicularis oculi muscle incision.

\section{Method of adding even levator advancement}

Levator advancement can also be performed together with the abovedescribed skin and OOM excision procedures. This both reduces the factors interrupting the levator action through the OOM, and has the following effects: formation of a CFS sling, Müller muscle tucking, and levator advancement (Fig. 6).

\section{Postoperative management}

Patients are instructed to apply an ice pack along with mild compression for 1 to 2 days. They are instructed to practice opening their eyes wide for 2-3 minutes after applying an ice pack for $5 \mathrm{~min}$ utes. On the 1st day after surgery, the above process is repeated if possible.

\section{DISCUSSION}

The author sought a way to correct ptosis by adding a surgical procedure to a method similar to existing non-incisional methods. In this surgical method, the effect in terms of correction of ptosis is so natural that it is difficult to measure precisely by the margin to reflex distance 1 (MRD1) alone. Therefore, ptosis correction was analyzed in terms of the change in eyebrow location in order to obtain accurate measurements of the outcomes. In a previous study, it was reported that when the eyebrow was pulled up, the MRD1 increased by about $18 \%$ [7]. Twelve patients who underwent 6-point two-loop surgery, where skin hooding and other variables were minimized (mean age, 28.6 years; 11 females and 1 
male), were selected and their MRD1 was measured. The increase in the MRD1 was $0.915 \mathrm{~mm}$ (standard deviation, $0.584 \mathrm{~mm}$ ). The eyebrows were $1.117 \mathrm{~mm}$ (standard deviation, $1.462 \mathrm{~mm}$ ) lower than before surgery and the increase in the MRD1 adjusted to account for eyebrow movement was $1.12 \mathrm{~mm}(0.915+1.117 \times 0.18$ $\mathrm{mm}$ ). Furthermore, 24 patients who underwent separate-loop surgery, where skin hooding and other variables were minimized (mean age, 27.58 years; 23 females and 1 male) were selected and in 41 of their eyes (one loop of 12, two loops of 29), the effect of the separate-loop surgical method was measured. The increase in the MRD1 after one-loop surgery was $0.831 \mathrm{~mm}$ (standard deviation, $0.585 \mathrm{~mm}$ ), and the eyebrows were $1.415 \mathrm{~mm}$ (standard deviation, $1.242 \mathrm{~mm}$ ) lower. The estimated increase in the MRD1 adjusted to account for eyebrow movement was $1.084 \mathrm{~mm}$. The increase in the MRD1 after two-loop surgery was $0.988 \mathrm{~mm}$ (standard deviation, $0.624 \mathrm{~mm}$ ), and the eyebrows were $1.658 \mathrm{~mm}$ (standard deviation, $1.195 \mathrm{~mm}$ ) lower. Thus, the estimated increase in the MRD1 adjusted to account for eyebrow movement was $1.283 \mathrm{~mm}$. Through such data, the rough ptosis correction effect of these surgical procedures can be estimated. In particular, better surgical results can be expected from those who have thinner and softer soft tissue, and if the skin is thick and tough, the effect can be relatively small.

In the author's experience, the method of surgery in which the loop is separated from the double eyelid line is more favorable for the correction of ptosis, as the loop can be tied more tightly and a natural line can be created separately. In the method in which a loop and line are connected (the continuous method), knotting the suture tightly for correcting ptosis makes the line thicker, thereby making a deep line and maintaining swelling for long time. However, in the latter method (the continuous method), the direction of the backward vector was connected upward with a single thread naturally. A double fold line was thus maintained well. Therefore, this method is useful in patients with a weakened line after surgery using an incisional method or with a double eyelid with a few lines. When combining a loop and double eyelid line, as it is appropriate for the size of the line to be similar to the tarsal plate, this technique yielded good results for double eyelids measuring 6-8 $\mathrm{mm}$. However, when a very big or very small double eyelid is needed, a separate line is recommended (Table 1).

The complications of this surgery include knot visibility, asymmetry, eye irritation (discomfort), ectropion, infection, and diplopia [8]. The treatment for each complication is as follows.

For loss of the fold crease or knot visibility, part of the OOM and septum below the slit where the knot will be made is excised. Here, a small pocket is made in order for the knot to easily placed below the OOM. When making knots, they should be made to be pulled in the posterior direction (i.e., placed deeply into the tissue) if possible. If the knot is placed deeply into the tissue posteriorly, the knots below the OOM are not visible, and as the suture can be cut with 2
Table 1. Comparison between the continuous and separate methods

\begin{tabular}{ll}
\hline Continuous method & \multicolumn{1}{c}{ Separate method } \\
\hline Simple, fast & Complicated \\
Ptosis tension $\rightarrow$ fold tension & Separate tension \\
6-8 mm fold height & More flexible fold height \\
Swelling is long-lasting & Fast recovery \\
$\begin{array}{c}\text { Good for multiple folds, sec- } \\
\text { ondary blepharoplasty }\end{array}$ & $\begin{array}{c}\text { Natural, fast recovery, shallow fold, good for } \\
\text { very high or low folds }\end{array}$ \\
$\begin{array}{c}\text { Suitable for patients who do } \\
\text { not want knots to be untied }\end{array}$ & $\begin{array}{c}\text { Good for who need more ptosis correction } \\
\text { Suitable for patients who do not need a fold } \\
\text { (i.e., already have a fold) }\end{array}$ \\
\hline
\end{tabular}

mm remaining from the knots, the odds of the knots coming untied can be reduced. If granuloma appears around the knots, a large lump can develop, but it improves once the knots are removed. Such a granuloma often occurs as a result of incidental contamination by powder from a medical glove or gauze fibers. Caution should therefore be taken to avoid these possibilities.

Slight asymmetry often occurs. To reduce asymmetry, the skin design should be made accurately. As the size of the tarsal plate is similar on both sides, if the needle is adjusted to exit from the superior margin of the tarsal plate when it exits from the conjunctiva, the asymmetry can be reduced. Asymmetry can occur when levator function, eyebrow movement, and eye protrusion are different. In such cases, the design for both sides should be made differently, or correction of ptosis should be done through a stitch method.

For cornea irritation and discomfort, the suture should not be exposed by more than 1-2 $\mathrm{mm}$ from the conjunctiva. If a patient complains of ocular discomfort immediately after surgery, the inside of the eye should be checked immediately and if there is extensive exposure of the suture, the knot should be undone and the double eyelid remade. If it is difficult to check the suture, eye ointment should be used for 1 or 2 days. Then, the decision to untie the knots is made based on an assessment of whether the irritation has continued.

Ectropion or an excessively deep crease can occur early if strong knots are made. However, they improve over time.

Diplopia can occur as an early complication, as the levator muscle and superior rectus muscle are connected to each other. Diplopia usually resolves within 1-2 weeks.

This paper was written to share the experiences of the author, with the hope of contributing to the development of better methods in the future.

\section{NOTES}

\section{Conflict of interest}

No potential conflict of interest relevant to this article was reported. 


\section{ORICD}

Tae Joo Ahn https://orcid.org/0000-0003-2892-446X

\section{Supplemental material}

Supplementary materials can be found via https://doi.org/10.14730/ aaps.2019.01879

\section{REFERENCES}

1. Ahn TJ. Blepharoptosis correction with stitch method. J Korean Soc Aesthetic Plast Surg 2010;16:167-70.

2. Neligan PC. Plastic surgery. In: Kemigan CL, Vidal EC, Pusic AL, et al. editors. Evidence-based medicine and health services research in plastic surgery. 3rd ed. London: Elsevier; 2013. p. 163-8.

3. Ahn TJ, Kim JH, Lee EI, et al. Nonincisional conjoint fascial sheath suspension: a novel technique for minimally invasive blepharoptosis correction. Ann Plast Surg 2017;79:334-40.

4. Suzie HC, William PC, Cho IC, et al. Comprehensive review of asian cosmetic upper eyelid oculoplastic surgery: Asian blepharoplasty and the like. Arch Aesthetic Plast Surg 2014;20:129-39.

5. Ahn TJ, Kim K. Mild ptosis correction with stitch method. Arch Aesthetic Plast Surg 2012;18:15-20.

6. Lee EI, Ahn TJ. Mild ptosis correction with the stitch method during incisional double fold formation. Arch Plast Surg 2014;41:71-6.

7. Lee EI, Kim NH, Park RH, et al. The relationship between eyebrow elevation and height of the palpebral fissure: should postoperative brow descent be taken into consideration when determining the amount of blepharoptosis correction? Arch Aesthetic Plast Surg 2014;20:20-5.

8. Korean Society for Aesthetic Plastic Surgery. Aesthetic plastic surgery. Seoul: Koonja Publishing; 2016. 